\title{
Hyaluronic Acid Intra-Articular Injections in Patients Affected by Moderate to Severe Glenohumeral Osteoarthritis: A Prospective Randomized Study
}

\author{
G. Di Giacomo ${ }^{1} \quad$ N. de Gasperis ${ }^{1}$ \\ ${ }^{1}$ Department of Orthopedics, Concordia Hospital for "Special \\ Surgery," Rome, Italy \\ Joints 2017;5:138-142.
}

Address for correspondence N. de Gasperis, MD, Department of Orthopedics, Concordia Hospital for "Special Surgery," Via delle Sette Chiese 90, Rome 00145, Italy (e-mail: nicola.degasperis@gmail.com).

\begin{abstract}
Purpose The aim of this study was to evaluate the efficacy and safety of intra-articular injection treatment with high molecular weight HA (Hyalubrix, $30 \mathrm{mg} / 2 \mathrm{~mL}$, molecular weight $>1,500 \mathrm{kDa}$ ) in patients affected by moderate to severe glenohumeral osteoarthritis (OA).

Methods Seventy-eight patients, affected by shoulder OA grade II-IV were randomized in two groups. Patients included in case group were treated with three intraarticular injections of HA and a specific physiotherapy program, whereas patients included in the control group received the only physical therapy. The follow-up examination was 6 months for both groups. The evaluation of functional status of treated shoulder, range of motion, and pain was performed before treatment and at the final follow-up examination by means of the Constant score. The safety evaluation of the treatment was also performed recording any adverse events.

Results Statistical analysis revealed a significant difference $(p<0.05)$ between the two groups in terms of pain reduction and improvement in the activities of daily living. In particular, case group subjects affected by grade III and IV OA had a significant

Keywords

- hyaluronic acid

- injection

- glenohumeral

- osteoarthritis

- shoulder improvement in the Constant score (18.2 \pm 5.4 and $19.2 \pm 5.9$, respectively).

Conclusion This study showed that the combination of intra-articular injection of hyaluronic acid (Hyalubrix, $30 \mathrm{mg} / 2 \mathrm{~mL}$ ) with physical therapy program was more effective in comparison with the only physical therapy in reducing pain in patients affected by glenohumeral OA.

Level of Evidence Level II, randomized controlled study.
\end{abstract}

\section{Introduction}

Glenohumeral osteoarthritis (OA) is characterized by the appearance of typical degenerative changes in the cartilage, synovial membrane, synovial fluid, and subchondral bone. ${ }^{1} \mathrm{OA}$ is one of the main reasons of persistent shoulder pain and reduced range of motion (ROM) $)^{2,3}$ and can compromise the activities of work and daily living leading to the development of depressive syndromes. ${ }^{1,4}$ The treatment of this pathology can be conservative in patients affected by grade I-III OA or grade IV OA with contraindications to surgical treatment. The conservative approach generally includes intra-articular injections with corticosteroids, hyaluronic acid (HA), physical therapy, or the use of oral analgesics. ${ }^{5-11}$ However, analgesics and nonsteroidal anti-inflammatory drugs (NSAIDs) are often associated with essential side effects. ${ }^{12-14}$ Viscosupplementation with HA intra-articular injections is a nonsurgical treatment that can improve pain relief and lead to a partial recovery
DOI https://doi.org/ $10.1055 / \mathrm{s}-0037-1605389$. ISSN $2282-4324$
Copyright @ 2017 Georg Thieme Verlag KG Stuttgart · New York
License terms

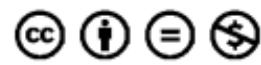


of glenohumeral joint ROM. Many studies have demonstrated that HA intra-articular injections may improve the cartilage homeostasis having protective effects on the joint cartilage in this degenerative pathology, ${ }^{15-28}$ especially in knee OA. ${ }^{29-33}$ However, the efficacy and safety of HA intra-articular injection treatment in glenohumeral OA needs to be further investigated, since only few studies have evaluated the efficacy and safety of HA in OA of the shoulder. $8,17,21,34-36$

The objective of this randomized prospective open-label clinical trial was to evaluate the efficacy and safety of intraarticular injection treatment with high-molecular-weight HA in patients affected by moderate to severe glenohumeral $\mathrm{OA}$ in terms of pain reduction and improvements of limited ROM. The hypothesis of the study was that HA intra-articular injections in patients affected by moderate to severe glenohumeral OA is a safe procedure that can reduce shoulder pain and improve glenohumeral ROM.

\section{Methods}

\section{Study Design}

This randomized controlled prospective open-label monocentric study was designed to determine whether intraarticular injections of HA (Hyalubrix, $30 \mathrm{mg} / 2 \mathrm{~mL}$, molecular weight $>1,500 \mathrm{kDa}$, Fidia Farmaceutici S.p.A.) in patients affected by grade II-IV shoulder OA provide significant reduction in pain during activity at up to 6 months.

\section{Participants}

Seventy-eight patients affected by grade II and III OA and grade IV shoulder OA with contraindications to surgical treatment were considered for the purpose of this study and followed for 6 months. Local ethics committee approval (Concordia Hospital for Special Surgery Rome Ethical Committee approval no. 3/2014) and written informed consent were obtained prior to the patient's enrolment, and the study was conducted in accordance with the Helsinki Declaration of 1975 and subsequent revisions.

The criteria for the inclusion in the study were: patients with good general health status older than 50 years, radiographic confirmation of grade II or III shoulder OA or grade IV shoulder OA with contraindications to surgical treatment, chronic shoulder pain for at least 6 months but less than 2 years, and limitations in ROM.

Reasons for exclusion from the study were: concomitant rotator cuff tears, adhesive capsulitis, previous shoulder surgery, previous humeral head fractures, chondrocalcinosis, cervical spine disorders, metabolic diseases, and poor general health status. Furthermore, patients with grade I OA and those with grade IV OA with surgical indications (shoulder arthroplasty) were not included in the study. All analgesics, bisphosphonates, and oral pharmaceutical products containing glucosamine and chondroitin sulfate had to be discontinued 15 days prior to the beginning of treatment and baseline assessment.

\section{Interventions}

Patients were randomly allocated to two different homogeneous groups. Thirty-nine patients for the first group (case group) and 39 patients for the second group (control group) were recruited. The sample was systematically randomized; for every two patients, the assignment criteria were inverted to allocate the patients uniformly to two different groups.

The treatment for patients belonging to the case group consisted of three intra-articular injections with HA (molecular weight $>1,500 \mathrm{kDa}$ ) and one injection every 15 days combined with a specific physiotherapy program. The intraarticular injections were performed by using a posterior shoulder approach. Patients belonging to the control group were treated only with physical therapy.

The physical therapy program was the same for both groups. It was performed with a professional therapist, had a 3-month duration with a frequency of 3 days every week, and started for both groups the day after the first medical examination. It consisted of passive capsular stretching for recovery of ROM, isometric exercises for deltoid, rotator cuff, and scapulothoracic muscles, isotonic exercises for scapulothoracic muscles (closed kinetic chain), and hydrokinesis therapy.

\section{Outcome Measures}

The follow-up examination was performed at 6 months from the beginning of the therapy for both groups. The evaluation of functional status of treated shoulder, ROM, and pain was performed before treatment and at the final follow-up examination by means of the Constant score. The safety evaluation of intra-articular injection with Hyalubrix (30 mg/2 mL) was further performed recording any adverse events (AEs) experienced by the patients during the study.

\section{Statistical Analysis}

Sample size calculation was based on the expected difference between groups in the primary outcome (Constant score). According to data obtained in our previous study, ${ }^{35}$ setting $\alpha$ value equal to 0.05 and minimum accepted power of $80 \%$, we retained the sample of 78 patients (39 for each group) appropriate for this study.

Statistical analysis was performed by STATISTICA 7.0 software (StatSoft Inc, Tulsa, Oklahoma, United States). Outcome variables (forward elevation, external rotation, and Constant score) were considered as continuous, and distribution of subjects along the three variables verged on normality ( $p>0.05$; Shapiro-Wilk $=0.958,0.975$, and 0.946, respectively); therefore, a one-way ANOVA for repeated measures was performed. Post hoc tests (Tukey's test, $p<0.05$ ) were scheduled between the two groups and within the same group. Afterward, a subgroup analysis was performed within the case group according to the severity of OA (16 patients with grade II, 14 with grade III, and 9 with grade IV). In this way, a one-way ANOVA for independent groups was performed. The treatment safety was assessed through descriptive statistics of vital signs and summary of AEs.

\section{Results}

Of the 39 patients belonging to the case group, 17 were males and 22 were females. The mean age of the patients was $71.3 \pm 6.7$ years. There were 31 right shoulders and 8 left 
shoulders. The dominant side was treated in 28 patients (71.8\%). All patients referred shoulder pain since many months (average $11.4 \pm 4.8$ months); 25 of the patients $(64.1 \%)$ had moderate pain and 14 (35.9\%) had severe pain; none of them had mild pain; 10 (25.6\%) of our patients had been involved in trauma; and 29 (74.4\%) of the patients had a spontaneous pain. All the patients showed signs of OA at the X-ray examination: grade II in 16 (41\%), grade III in 14 (35.9\%), and grade IV in 9 (23.1\%) OA.

Of the 39 patients belonging to the control group, 15 were males and 24 were females. The mean age of the patients was $69.8 \pm 6.4$ years. There were 29 right shoulders and 10 left shoulders. The dominant side had been treated in 31 patients (79.5\%). All patients referred shoulder pain for several months (average $10.2 \pm 3.8$ months); 4 of the patients (10.3\%) had mild pain, 27 (69.2\%) had moderate pain, and 8 (20.5\%) had severe pain; 9 (23.1\%) of our patients had been involved in trauma, and 30 (76.9\%) of the patients had a spontaneous pain. All the patients showed signs of OA at the X-ray examination: 18 (46.1\%) had grade II, 14 (35.9\%) had grade III, and 7 (17.9\%) had grade IV OA. No significant differences were observed between the two groups for baseline characteristics.

Means and standard deviations of the outcome measures for each group, before and after treatment, are reported in -Table 1. There were no significant differences between the two groups before the treatment. Constant score, forward elevation, and external rotation variables were similar in the two groups at the baseline evaluation $(p>0.05)$. The ANOVA analysis for repeated measures for Constant score revealed a significant effect of the treatment between the groups $\left(F_{1.76}=7.885, p<0.05\right)$ and within groups $\left(F_{1.76}=15.735\right.$, $p<0.05)$. Regarding the forward elevation, the ANOVA analysis for repeated measure depicted a significant effect only within groups $\left(F_{1.76}=6.611, p<0.05\right)$, suggesting that there was a significant effect of the treatment along the time but not between the type of treatment $\left(\mathrm{F}_{1.76}=0.655, p>0.05\right)$. Differently, about the external rotation, the statistical analysis did not show a significant difference between the groups $\left(\mathrm{F}_{1.76}=1.061, p>0.05\right)$ nor within the groups $\left(\mathrm{F}_{1.76}=0.835\right.$, $p>0.05)$.

The one-way ANOVA performed only for the case group subjects revealed a significant effect of the treatment $\left(F_{2.36}=7.829, p<0.05\right)$. In particular, patients affected by grade III and IV OA had a significant improvement in the

Table 1 Means and standard deviations for case and control groups before $\left(t_{0}\right)$ and after $\left(t_{1}\right)$ treatment

\begin{tabular}{|l|l|l|}
\hline & Control group & HA group \\
\hline Constant score $_{\mathrm{t} 0}$ & $67.1 \pm 15.1$ & $71.9 \pm 12.1$ \\
\hline Constant score $_{\mathrm{t} 1}$ & $77.3 \pm 13.8$ & $88.1 \pm 9.3^{\mathrm{a}}$ \\
\hline Elevation $_{\mathrm{t} 0}$ & $137.9 \pm 21.5$ & $138.6 \pm 16.7$ \\
\hline Elevation $_{\mathrm{t} 1}$ & $146.8 \pm 19.8$ & $152.8 \pm 15.9$ \\
\hline External rotation $_{\mathrm{t} 0}$ & $24.2 \pm 6.2$ & $22.3 \pm 6.9$ \\
\hline External rotation $_{\mathrm{t} 1}$ & $26.4 \pm 7.9$ & $25.1 \pm 7.1$ \\
\hline
\end{tabular}

Note: to Baseline evaluation; ${ }_{t 1}$ Follow-up evaluation.

${ }^{a} p<0.05$; post hoc Tukey's test.

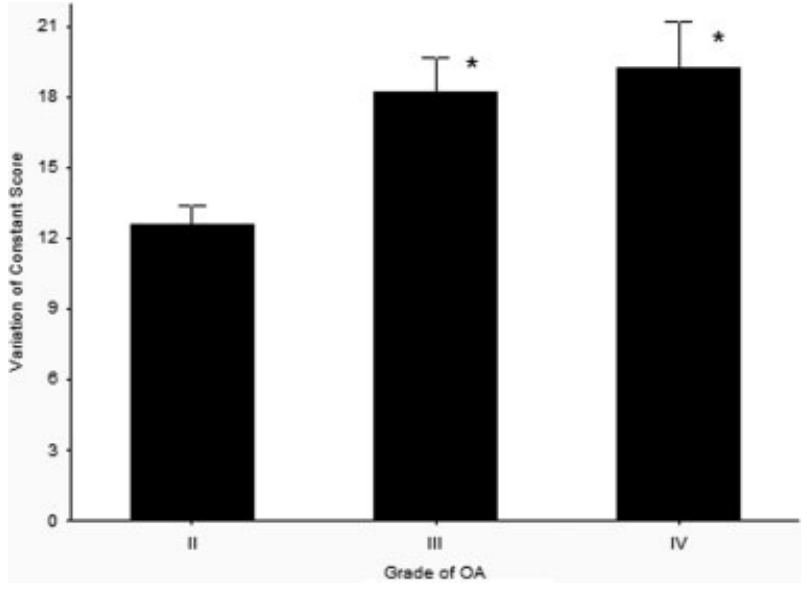

Fig. 1 Variation of Constant score (y-axis) after treatment in the case group, according to severity of OA (bars indicate the standard error). Grade III and IV OA showed a significantly higher variation compared with grade II ( ${ }^{*} p<0.05$ post hoc Tukey's test).

Constant score (18.2 \pm 5.4 and $19.2 \pm 5.9$, respectively). On the other hand, patients affected by grade II OA had no significant improvement in the Constant score $(p<0.05$ at the post hoc Tukey's test, - Fig. 1).

\section{Discussion}

Glenohumeral OA is a progressive disease that can compromise the activities of work and daily living because of its symptoms such as pain and reduction of ROM. Therefore, nonsurgical treatments are directed toward reducing symptoms and improving joint function.

The use of HA in patients affected by shoulder OA is described in literature in a few studies. Kwon et al, ${ }^{17}$ in a multicenter, randomized, double-blind, placebo-controlled trial, showed that only patients without concomitant shoulder pathologies, treated with intra-articular injections of HA, had a significant improvement in terms of pain. Similarly, Porcellini et $\mathrm{al}^{34}$ and Di Giacomo et $\mathrm{al}^{35}$ in their prospective studies, showed that HA treatment for shoulder OA significantly decreased pain and improved shoulder function for up to 6 months from the first injection. Also, Merolla et al, ${ }^{21}$ in a retrospective controlled trial, found that patients affected by mild to moderate glenohumeral OA had significant pain reduction and satisfaction, for up to 6 months, after treatment with intra-articular injections of HA. Silverstein et $\mathrm{al}^{36}$ found a significant improvement of shoulder pain in patient treated with 3 weekly HA intra-articular injections and no adverse events were observed. On the other hand, Blaine et $\mathrm{al}^{8}$ and Colen et $\mathrm{al}^{37}$ indicated that sodium hyaluronate is effective and well tolerated for the treatment of $\mathrm{OA}$ and persistent shoulder pain, but significant improvement in terms of shoulder pain was not achieved.

The purpose of the study was to evaluate the efficacy and safety of intra-articular injections with high-molecularweight HA (Hyalubrix) in patients affected by moderate to severe glenohumeral OA. To achieve this, two different kinds of treatments were compared: three intra-articular injections 
with HA (Hyalubrix, $30 \mathrm{mg} / 2 \mathrm{~mL}$, molecular weight $>1,500$ $\mathrm{kDa}$ ) associated with physical therapy rehabilitation versus the only physical therapy treatment. This open-label study on 78 patients showed a significant difference in pain relief $(p<0.05)$ between the two groups of patients in favor of HA. This demonstrated that patients affected by grade II-IVOA, who received HA intra-articular injections treatment in association with physical therapy, had a greater positive effect in terms of pain reduction compared with patients who received the only physical therapy treatment. Differently, this study did not show a significant difference in terms of ROM, between the two different types of treatment. Moreover, the reduction of pain led to an improvement in glenohumeral function and activity of daily living. This was due to the specific characteristics of HA able to restore synovial fluid properties in this degenerative disease. ${ }^{15-28}$

Results of the study also showed a significant difference within the case group. In particular, patients affected by grade III and IV OA had a significant pain reduction, whereas patients affected by grade II OA had an improvement in pain, but it was not significant. This could be due to the molecular weight of Hyalubrix ( $>1,500 \mathrm{kDa}$ ) that also has an important mechanical effect.

Thanks to the efficacy of HA treatment and to the absence of side effects or adverse events, we can confirm that three intra-articular injections of Hyalubrix can be considered a safe and effective treatment option for the management of shoulder pain due to moderate to severe glenohumeral OA. However, further prospective randomized controlled trials are necessary to provide exhaustive evidence of the longterm efficacy of HA-derived products in the treatment of glenohumeral OA in a larger population.

In conclusion, this study demonstrates the greater and long-lasting efficacy and safety of an intra-articular injection treatment with $\mathrm{HA}$ (Hyalubrix, $30 \mathrm{mg} / 2 \mathrm{~mL}$ ) combined with a physical therapy program in patients affected by moderate to severe glenohumeral $\mathrm{OA}$ in terms of pain relief and function improvement, compared with alternative treatments such as only physical therapy.

\section{References}

1 Nakagawa Y, Hyakuna K, Otani S, Hashitani M, Nakamura T. Epidemiologic study of glenohumeral osteoarthritis with plain radiography. J Shoulder Elbow Surg 1999;8(06):580-584

2 Chakravarty K, Webley M. Shoulder joint movement and its relationship to disability in the elderly. J Rheumatol 1993;20 (08):1359-1361

3 Steinfeld R, Valente RM, Stuart MJ. A commonsense approach to shoulder problems. Mayo Clin Proc 1999;74(08):785-794

4 Memel DS, Kirwan JR, Sharp DJ, Hehir M. General practitioners miss disability and anxiety as well as depression in their patients with osteoarthritis. Br J Gen Pract 2000;50(457):645-648

5 Andrews JR. Diagnosis and treatment of chronic painful shoulder: review of nonsurgical interventions. Arthroscopy 2005;21(03): 333-347

6 Calis M, Demir H, Ulker S, Kirnap M, Duygulu F, Calis HT. Is intraarticular sodium hyaluronate injection an alternative treatment in patients with adhesive capsulitis? Rheumatol Int 2006; 26(06):536-540
7 Carfagno DG, Ellenbecker TS. Osteoarthritis of the glenohumeral joint: nonsurgical treatment options. Phys Sportsmed 2002; 30(04):19-30

8 Blaine T, Moskowitz R, Udell J, et al. Treatment of persistent shoulder pain with sodium hyaluronate: a randomized, controlled trial. A multicenter study. J Bone Joint Surg Am 2008; 90(05):970-979

9 Brander VA, Gomberawalla A, Chambers M, Bowen M, Nuber G. Efficacy and safety of hylan G-F 20 for symptomatic glenohumeral osteoarthritis: a prospective, pilot study. PM R 2010;2(04):259-267

10 Itokazu M, Matsunaga T. Clinical evaluation of high-molecularweight sodium hyaluronate for the treatment of patients with periarthritis of the shoulder. Clin Ther 1995;17(05):946-955

11 Ramonda R, Franceschini M, Leardini G. Treatment of shoulder periarthritis with hyaluronic acid. Riv Ital Biol Med 1998;51 (2 Suppl): 1

12 American College of Rheumatology Subcommittee on Osteoarthritis Guidelines. Recommendations for the medical management of osteoarthritis of the hip and knee: 2000 update. Arthritis Rheum 2000;43(09):1905-1915

13 Singh G. Recent considerations in nonsteroidal anti-inflammatory drug gastropathy. Am J Med 1998;105(1B):31S-38S

14 Jordan KM, Arden NK, Doherty M, et al; Standing Committee for International Clinical Studies Including Therapeutic Trials ESCISIT. EULAR Recommendations 2003: an evidence based approach to the management of knee osteoarthritis: Report of a Task Force of the Standing Committee for International Clinical Studies Including Therapeutic Trials (ESCISIT). Ann Rheum Dis 2003;62 (12):1145-1155

15 Sinusas K. Osteoarthritis: diagnosis and treatment. Am Fam Physician 2012;85(01):49-56

16 Balazs EA, Denlinger JL. Viscosupplementation: a new concept in the treatment of osteoarthritis. J Rhematol Suppl 1993;39:3-9

17 Kwon YW, Eisenberg G, Zuckerman JD. Sodium hyaluronate for the treatment of chronic shoulder pain associated with glenohumeral osteoarthritis: a multicenter, randomized, double-blind, placebo-controlled trial. J Shoulder Elbow Surg 2013;22(05): 584-594

18 Colen S, Haverkamp D, Mulier M, van den Bekerom MP. Hyaluronic acid for the treatment of osteoarthritis in all joints except the knee: what is the current evidence? BioDrugs 2012;26(02): 101-112

$19 \mathrm{Gu}$ Q Zhou N, Shanghai J. Research progress of viscosupplementation [Article in Chinese]. Zhongguo Xiu Fu Chong Jian Wai Ke Za Zhi 2011;25(06):755-760

20 Abate M, Pulcini D, Di Iorio A, Schiavone C. Viscosupplementation with intra-articular hyaluronic acid for treatment of osteoarthritis in the elderly. Curr Pharm Des 2010;16(06):631-640

21 Merolla G, Sperling JW, Paladini P, Porcellini G. Efficacy of Hylan G-F 20 versus 6-methylprednisolone acetate in painful shoulder osteoarthritis: a retrospective controlled trial. Musculoskelet Surg 2011;95(03):215-224

22 Abatangelo G, O'Regan M. Hyaluronan: biological role and function in articular joints. Eur J Rheumatol Inflamm 1995;15:9-16

23 Altman RD, Moskowitz R; Hyalgan Study Group. Intraarticular sodium hyaluronate (Hyalgan) in the treatment of patients with osteoarthritis of the knee: a randomized clinical trial.J Rheumatol 1998;25(11):2203-2212

24 Carrabba M, Paresce E, Angelini M, Re KA, Torchiana EEM, Perbellini A. The safety and efficacy of different dose schedules of hyaluronic acid in the treatment of painful osteoarthritis of the knee with joint effusion. Eur J Rheumatol Inflamm 1995; 15:25-31

25 Maheu E. Hyaluronan in knee osteoarthritis: a review of clinical trials with Hyalgan. Eur J Rheumatol Inflamm 1995;15:17-24

26 Kotz R, Kolarz G. Intra-articular hyaluronic acid: duration of effect and results of repeated treatment cycles. Am J Orthop 1999; 28(11, Suppl):5-7 
27 Frizziero L, Govoni E, Bacchini P. Intra-articular hyaluronic acid in the treatment of osteoarthritis of the knee: clinical and morphological study. Clin Exp Rheumatol 1998;16(04):441-449

28 Listrat V, Ayral X, Patarnello F, et al. Arthroscopic evaluation of potential structure modifying activity of hyaluronan (Hyalgan) in osteoarthritis of the knee. Osteoarthritis Cartilage 1997;5(03): 153-160

29 Migliore A, Tormenta S, Martin Martin LS, et al. The symptomatic effects of intra-articular administration of hylan G-F 20 on osteoarthritis of the hip: clinical data of 6 months follow-up. Clin Rheumatol 2006;25(03):389-393

30 Stitik TP, Blacksin MF, Stiskal DM, et al. Efficacy and safety of hyaluronan treatment in combination therapy with home exercise for knee osteoarthritis pain. Arch Phys Med Rehabil 2007;88 (02):135-141

31 Phiphobmongkol V, Sudhasaneya V. The effectiveness and safety of intra-articular injection of sodium hyaluronate (500-730 kDa) in the treatment of patients with painful knee osteoarthritis. J Med Assoc Thai 2009;92(10):1287-1294

32 Foti C, Cisari C, Carda S, et al. A prospective observational study of the clinical efficacy and safety of intra-articular sodium hyalur- onate in synovial joints with osteoarthritis. Eur J Phys Rehabil Med 2011;47(03):407-415

33 Vetro A, Iovane A, Di Gesu M, Giordan N, Mantia F, Mantia R. Pain relief and functional recovery over a six-month period after intraarticular injection with sodium hyaluronate (Mw 1500 - 2000 Kda) in osteoarthritis of the knee. Eur J Musculoskel Dis 2014; 3:25-33

34 Porcellini G, Merolla G, Giordan N, et al. Intra-articular glenohumeral injections of HYADD ${ }^{4} 4-G$ for the treatment of painful shoulder osteoarthritis: a prospective multicenter, open-label trial. Joints 2016;3(03):116-121

35 Di Giacomo G, De Gasperis N. The role of hyaluronic acid in patients affected by glenohumeral osteoarthritis. J Biol Regul Homeost Agents 2015;29(04):945-951

36 Silverstein E, Leger R, Shea KP. The use of intra-articular hylan G-F 20 in the treatment of symptomatic osteoarthritis of the shoulder: a preliminary study. Am J Sports Med 2007;35(06):979-985

37 Colen S, Geervliet P, Haverkamp D, Van Den Bekerom MP. Intraarticular infiltration therapy for patients with glenohumeral osteoarthritis: a systematic review of the literature. Int J Shoulder Surg 2014;8(04):114-121 